\title{
Influence of the proprioceptive neuromuscular facilitation exercise programs on idiopathic scoliosis patient in the early 20 s in terms of curves and balancing abilities: single case study
}

\author{
Byung-Ki Lee* \\ Department of Physical Therapy, Daewon University College, Jecheon, Korea
}

The purpose of this study was to determine the influence of the proprioceptive neuromuscular facilitation (PNF) exercise programs for idiopathic scoliosis with a female patient in the early 20s in terms of her spinal curve and balancing abilities. The study subject was selected among 21-year-old female college students. There were no particular activities that the subject could not perform, but patient complained of difficulty in maintaining the standing position for a prolonged time. $\mathrm{Pa}$ tient chest $X$-ray results showed S-shaped curves tilted towards the left or right in the lumbar spine, thoracic spine, and cervical spine areas. The PNF exercise programs consists of seven therapeutic exercise programs, including home exercises considering the patients' balancing abilities, the lateral symmetry of the spinal sway, the distortion, and the height of the pelvis and scapula bones. The programs last for 6 weeks and includes three sessions per week, with each session lasting for $30 \mathrm{~min}$. Before and after the execution of the PNF exercise programs, the subject was monitored for the changes in her spinal sways through chest X-ray tests. Also, using a balance measurement instrument, the subject's static and dynamic balancing abilities were tested. After executing the PNF exercise programs for 6 weeks, the spinal sways of the subject were corrected, and her static and dynamic balancing abilities were improved compared to the baseline values.

Keywords: Idiopathic scoliosis, Proprioceptive neuromuscular facilitation, Closed kinetic chain exercise, Balance ability

\section{INTRODUCTION}

Spinal scoliosis is defined as a condition in which the alignment of the spine at one or two points is swaying laterally from the central axis of the human body, or is rotated. Idiopathic scoliosis, which occurs without any clear reason, is a condition where there is a structural change in Cobb angle by at least 10 degrees (Bunnell, 1986).

The symptoms of scoliosis usually do not include pain as it progresses. Moreover, due to the abnormal posture during the teenage years and beyond, the patients experience distortion in the hip joints, spinal alignment, and tilted head. Also, scoliosis causes a balance problem in the standing and sitting positions, making

the individual feel fatigued when he/she has been standing or sitting for a prolonged time, or otherwise limiting the individual's daily life activities. In a severe case of scoliosis, the muscular force and flexibility of the body can be affected, triggering pain in the spine. In the case of young females, this disease may also cause cosmetic problems, such as imbalance in the height of the shoulders and the pelvis, inviting further psychological or social problems (Srivastava et al., 2016). Idiopathic scoliosis normally progresses without pain, and its cause is unclear and the effectiveness of the treatment is uncertain. Until now, the most recommended therapy in hospitals is to use a support tool depending on the angle of scoliosis or surgery. It has been reported that if the disease is discovered earlier, exercise and support-wearing may alleviate the

${ }^{*}$ Corresponding author: Byung-Ki Lee (ib) http://orcid.org/0000-0002-1639-3318 Department of Physical Therapy, Daewon University College, 316 Daehak-ro, Jecheon 27135, Korea

Tel: +82-43-649-3224, Fax: +82-43-649-3690, E-mail: toppt@naver.com

Received: September 25, 2016 / Accepted: December 1, 2016 
symptom without surgery (Landauer et al., 1997).

Proprioceptive neuromuscular facilitation (PNF), which was developed by Knott, a physical therapist, and Kabat, a doctor, in the 1940s, is an exercise that stimulates the movements of the body in a diagonal direction, and that enables muscle contraction when there are paralyzed muscles or pain during physical movement (Bae, 1993). PNF, in theory, is a philosophy as well as a set of basic principles and procedures. One of the pillars of the PNF principles, "positive approach," is to use the movement of body segments as well as manual resistance, which do not trigger pain in a patient with pain and dysfunction in the muscular-skeletal system (Adler et al., 2002).

Therefore, this study was conducted to determine the influence of PNF on the sway of the spine and the balancing abilities of female idiopathic scoliosis patient in the early 20s, and to provide the basic data needed for the sports rehabilitation studies of young female scoliosis patient.

\section{MATERIALS AND METHODS}

\section{Participant}

The study subject was selected among the 21-year-old female patient who visited hospitals in Jecheon city and were diagnosed with scoliosis in March 2016 (Table 1). The subject was all college students, who were seated on a chair for more than 6 hours a day on average, listening to lectures. When she was in second year high school, she complained, after her dental correction, that the heights of both her shoulders began to become different, and that she felt that she was becoming more dependent on her right foot to support her weight. There were no specific daily life activities that she could not perform, but she complained of difficulty in maintaining the standing position for long.

The examination of the subject's chest X-ray images to see the lateral sways of her spines revealed that her cervical and thoracic spines were mildly swayed to the right side as a convex sway of the spine towards the right was observed. Moreover, the left-side scapular inferior angle was lower than the right-side inferior angle by $1.5 \mathrm{~cm}$, indicating that the positions of the scapular bones on the two sides were asymmetrical. The lumbar spines of the patient were significantly swayed in a convex way towards the left side,

Table 1. Characteristics of the participant

\begin{tabular}{lccccc}
\hline Sex & Age $(\mathrm{yr})$ & Height $(\mathrm{cm})$ & Weight $(\mathrm{kg})$ & Diagnosis & $\begin{array}{c}\text { Rehabilitation } \\
\text { period }\end{array}$ \\
\hline Female & 21 & 163 & 46 & Scoliosis & 6 weeks \\
\hline
\end{tabular}

and the spinous process of the lumbar spine, when observed from behind the subject, was facing the right side. Therefore, in the anterior view of the subject, it was twisted towards the left side, causing an abnormal alignment of the spine. The right side of the pelvis was also higher than the left side (Fig. 1). The subject did not complain of pain in her daily life, but she confessed that when she looked into a full-sized mirror, she felt stressed because the heights of the left and right shoulders looked different.

\section{Instruments}

The instrument that was used in this study to measure the balancing abilities of the patient was the balance-measuring instrument (BT-4; Hur Lab, Koarla, Finland), which could evaluate the static and dynamic balancing abilities (Piirainen et al., 2013). The balancing equipment that was used for the test had a $100-\mathrm{Hz}$ sampling rate and a rectangular shape. At the point of each segment was a strain gauge. The recommended positions of the feet were drawn on the surface of the rectangular platform to help the subject properly position herself. Four sensors were used to identify the center of pressure (COP) of each subject, and the perturbation over time was measured and analyzed using the Smart-Suit Balance software ver. 1.4 (manufacturer, city, country). Each subject was tested three times before the start of the exercise programs and 3 weeks after the completion of the exercise programs.

\section{Static balance test}

Each subject took the 30-sec-long static balance test in three sessions, with the eyes closed, and the average of the measurement values obtained was used for the analysis. For the positions of the feet, the heels of both feet were kept $2 \mathrm{~cm}$ apart from each other, with the shoes taken off, and the feet were turned 15 degrees out-
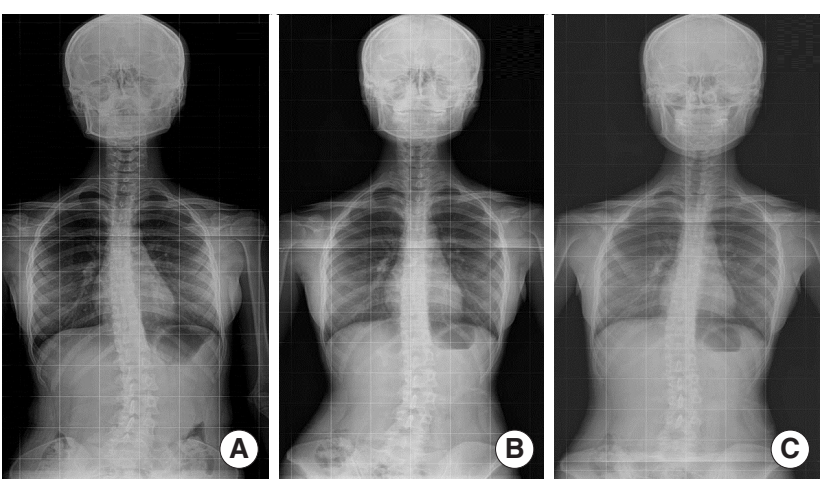

Fig. 1. Chest X-ray. (A) Exercise before the start of shooting (April 10, 2016); (B) Exercise 3 weeks elapsed after shooting (May 2, 2016); (C) Exercise 6 weeks elapsed after shooting (May 23, 2016). 
ward on both sides. The hands were placed naturally on the seam of the trouser (Borg and Laxåback, 2010). The parameters that were measured in the static balancing test were the length of the sway line, which indicated the movement of the posture due to the COP (trace length), and the value of the area $\left(\mathrm{mm}^{2}\right)$ of the trace line (C90A; COP 90\%-confidence-level ellipse area).

\section{Dynamic balance test}

For the dynamic balance test, the "limit of stability" method provided in the software (Smart unit 1.4) was used. With the socks off, the feet were positioned $2 \mathrm{~cm}$ apart from each other, and each foot was turned 15 degrees outward while both hands were placed naturally on the seam of the trouser. As for the test, while both feet were fixed on the platform, the subject who was to stand straight, tried to tilt her body as far as possible towards the front/ rear/left/right directions, and maintained the tilted positions for 8 sec each time for calculating the highest tilt level in each direction. D'Hondt et al. (2011) defined the dynamic balancing ability as the sum of the maximum tilt angle in the four directions, based on the data of the maximum length towards the front/rear/left/ right. In this study as well, the sums of the maximum tilt angles in the four directions before and after the exercise programs, respectively, were compared for analysis.

\section{Procedure}

This study was conducted in four stages. The first stage was the evaluation and planning of the subject, the second was the preliminary test, the third was the application of the PNF exercise programs, and the fourth was the conduct of follow-up tests. The application of the PNF exercise programs started on April 11, 2016 and continued over 6 weeks (18 sessions), until May 20, 2016. Each session lasted $30 \mathrm{~min}$. The tests were conducted on the subject, who gave her informed consent to participate in the study considering its purpose and duration.

\section{Evaluation of the subjects and treatment plan}

PNF is based on the hypothesis regarding the cause of impairment of the relevant body-structured body function for the improvement of the activity limitation being complained about by the patient, by including the international classification of functioning, disability, and health to improve the patient's daily-life-activity limitations. Based on this, before treating the study subject, an interview of each of them was conducted, and the subjective complaint was heard. The subject complained of difficulty in maintaining the standing position for a prolonged time in her daily life due to scoliosis, felt that her full body weight was being supported by her right foot, and had difficulty maintaining her balance while standing.

\section{Baseline and follow-up tests}

The baseline tests were performed on the day prior to the start of the exercise programs. The tests included chest X-ray imaging to see the change in the swaying of the spine, and static/dynamic balancing tests. The chest X-ray imaging was repeated 3 weeks after the completion of the exercise programs, and 6 weeks after the completion of all the exercise programs, the chest X-ray imaging and the dynamic/static balance tests were repeated in the same manner.

\section{PNF exercise programs}

The exercise programs in this study was composed of seven therapeutic exercise programs, including home exercises, and considering the balancing ability, lateral asymmetry of the sway, distortion, and heights of the pelvis and scapular bones, the exercise was executed with the initial posture with a wider bottom plane changed to one with a narrow bottom plane. Also, all the exercise programs were based on the basic principles of PNF, its procedures, and philosophies. Moreover, the PNF patterns and techniques were appropriately used. The PNF techniques that were used in the programs in this study also included the principle of combination of isotonics applied to the treatment of the patient. The PNF exercise programs, which were executed for a total of six weeks, consisted of three sessions a week, with each session lasting $30 \mathrm{~min}$. It combined two to three exercise programs in accordance with the level of adaptation of the patient to the exercises (Tables $2,3)$. Before beginning the exercise in earnest, the overall progress of the exercise and how to breathe deeply were explained to the subject. Also, when the subject complained of pain or fatigue, the exercise was suspended, and a 2- to 3-min break was given. (The break was excluded from the exercise time.)

\section{Data analysis}

The data collected from the test were visually presented for analysis. The results of the static balancing ability were shown in the sway lines indicating the movement of the posture due to the $\mathrm{COP}$ in the graphs showing the length $(\mathrm{mm})$ values and the area of the trace line drawn $\left(\mathrm{mm}^{2}\right)$. The dynamic balancing ability was shown by a graph indicating the maximum leaning angle in the front, rear, left, and right directions. 
Table 2. Proprioceptive neuromuscular facilitation programs

\begin{tabular}{|c|c|c|c|}
\hline Program No. & Major exercise content & Using PNF pattern & Patients position \\
\hline 1 & Stabilizing lumbo-pelvic systems & Deep breathing, pelvic posterior tilting & Hook-lying \\
\hline 2 & $\begin{array}{l}\text { Stabilizing lumbo-pelvic systems with correcting for spine alignment } \\
\text { (CKCE) }\end{array}$ & $\begin{array}{l}\text { Flexion-adduction-external rotation of left leg with flexion- } \\
\text { abduction-external rotation of left arm }\end{array}$ & Amphibian \\
\hline 3 & Correcting for spine alignment & $\begin{array}{l}\text { Bilateral leg flexion pattern to left with flexion-abduction- } \\
\text { external rotation of left arm }\end{array}$ & Supine \\
\hline 4 & Correcting for spine alignment with extremities (CKCE) & $\begin{array}{l}\text { Extension-abduction-internal rotation of right leg with } \\
\text { flexion-abduction-external rotation of left arm }\end{array}$ & Side-lying \\
\hline 5 & Correcting for spine alignment with pelvis \& extremities (CKCE) & $\begin{array}{l}\text { Anterior elevation of left pelvis with extension-abduction- } \\
\text { internal rotation of right arm }\end{array}$ & Sitting \\
\hline 6 & Correcting for spine alignment with pelvis \& extremities (CKCE) & $\begin{array}{l}\text { Anterior elevation of left pelvis with extension-abduction- } \\
\text { internal rotation of right arm }\end{array}$ & Standing \\
\hline 7 & Home exercise program & $\begin{array}{l}\text { Anterior elevation of left pelvis with extension-abduction- } \\
\text { internal rotation of right arm }\end{array}$ & Sitting \\
\hline
\end{tabular}

PMF, proprioceptive neuromuscular facilitation; CKCE, closed kinetic chain exercise.

Table 3. Exercise programs of each weeknum

\begin{tabular}{lcc}
\hline Weeknum & Program number & Duration (min) \\
\hline 1 & 1,2 & 30 \\
2 & $1,2,3$ & 30 \\
3 & $1,2,3$ & 30 \\
4 & $2,3,4$ & 30 \\
5 & $3,4,5$ & 30 \\
6 & $4,5,6$ & 30 \\
\hline
\end{tabular}

\section{RESULTS}

\section{Results of the chest radiography (X-ray)}

An X-ray imaging test was conducted before the start of the 6-week PNF exercise programs, upon its completion, and 3 weeks after its completion, totaling three tests. The examination of the lateral swaying of the spine in the baseline imaging test showed shallow leaning towards the right side of the cervical and thoracic spines. During the conduct of the PNF exercise programs, however, the alignment of the spine was restored close to the normal state, and the relatively more significant sway towards the left discovered in the lumbar spine was also restored to a better alignment after 3 weeks. The direction of the spinous process (towards the right side from the lumbar spine area) discovered during the baseline tests was also turned to the front, indicating that the distortion of the lumbar spine was also restored to a better alignment (Fig. 1).

\section{Results of the static balance test}

The researchers used BT- 4 (Hur Lab) to measure the trace length (TL) and the COP confidence ellipse area (C90A) to show the sway of the posture on the COP. With this test, it was shown
Table 4. Weight distribution

\begin{tabular}{llll}
\hline Eye condition & Time & Left & Right \\
\hline Eye open & Pre & 45.7 & 54.3 \\
& Post & 46.78 & 53.22 \\
Eye closed & Pre & 47.9 & 52.1 \\
& Post & 49.41 & 50.59 \\
\hline
\end{tabular}

that the test values obtained after the completion of the 6-week PNF exercise programs were lower than the baseline test values (Fig. 2). Moreover, with regard to the weight distribution between the two feet, the weight was leaning more towards the right foot in the baseline test but was more evenly distributed in the follow-up test (Table 4).

\section{Results of the dynamic balance test}

Using the limit of stability programs of BT-4, the researchers had the subject tilt her entire bodies towards the front/rear/right/ left directions, and asked them to maintain such tilted position for $8 \mathrm{sec}$. The measurement results of the leaning angle in all the directions showed that the values had increased in the follow-up test compared to the baseline test. During the baseline test, the maximum leaning angle, which was 2.72 degrees, increased to 4.13 degrees towards the right, indicating dynamic balancing ability symmetry approaching the limit of the leaning angle in which the balance can be maintained (5.35 degrees) (Fig. 3).

\section{DISCUSSION}

Idiopathic scoliosis is the state of the abnormal curve of the coronal plane and can be classified into types $\mathrm{C}$ and $\mathrm{S}$ depending on 

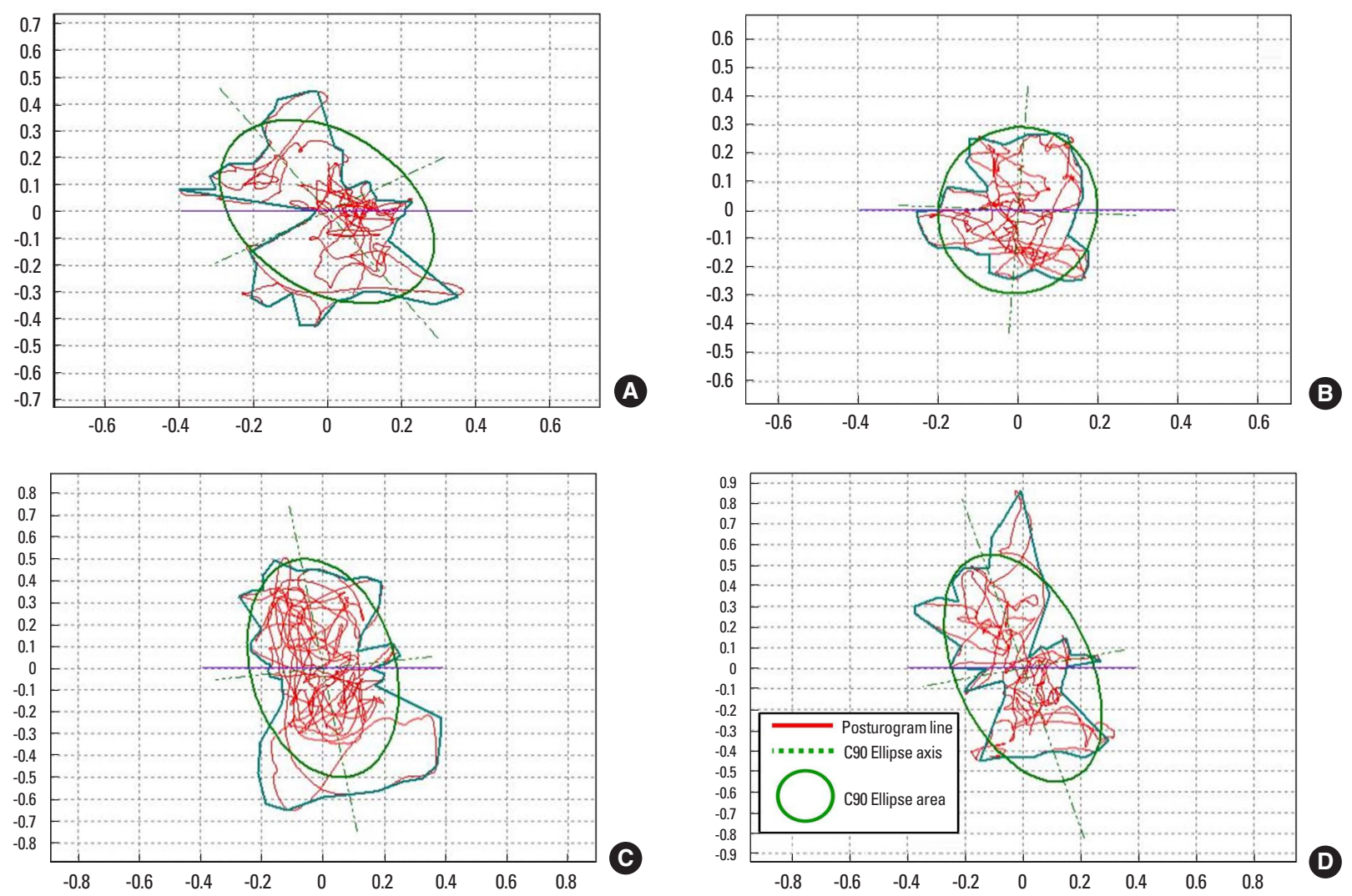

Fig. 2. Graph of static balance test outcomes: (A) pretest (eyes open); (B) posttest (eyes open); (C) pretest (eyes closed); (D) posttest (eyes closed).

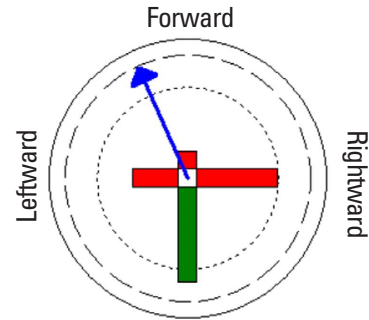

Rearward

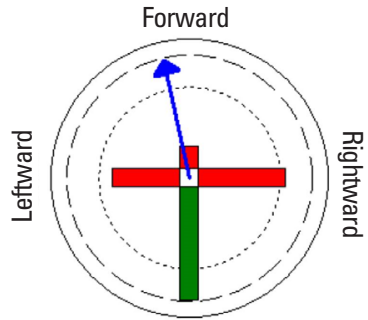

Rearward
B

$\bigcirc 8$ degrees $(7$ degrees 5 degrees $\bigcirc 8$ degrees $\quad 7$ degrees 5 degrees

Fig. 3. Graph of limit of stability for dynamic balance test outcomes: (A) pretest, (B) posttest.

the form. The normal spine is facing the convex surface, and the spinous process faces the concave surface, forming a multidimensional rotating feature (Pasha et al., 2016). Such transformation of the spine leads to the problem of posture balance (Yamada et al., 1969). Moreover, it is known that the swaying radius of the swaying area of the center of the body mass is much higher than that of a healthy person. The study subject was 21 -year-old female college students who had to spend much time sitting on her chair while listening to their teachers' lectures, had almost no time to exercise, and was assuming bad postures while she was seated. The X-ray image of one subject showed that as seen from the coronal plane, the lumbar spine had an abnormal sway towards the left and was concave towards the right (i.e., showing an abnormal spinal sway). On the sagittal plane, the spinous process of the lumbar spine was twisted towards the right. The cervical and thoracic spines showed a minor sway towards the right and was concave towards the left. As for the heights of the scapular bones and the pelvis on the transverse plane, the right-side scapular bone was higher than the left-side, and the same was true for the pelvis bone. As such, the subject was diagnosed with S-type scoliosis (Fig. 1). In the study conducted by Nault et al. (2002), the sway of the body center of a scoliosis patient was found to be higher than that of a healthy person. The researchers argued that the standing posture caused instability. In this study, the balancing measuring instrument called "BT-4" was used to measure the TL and the COP 90\%-confidence-level ellipse area of the sway of posture from the COP. The results were that all the figures that were obtained in the baseline test were high. In the standing posture, the weight was leaning 
more towards the right foot (Table 4; Figs. 2, 3). This meant that the static and dynamic balance of the scoliosis patient were not good. The static balance result indicated that the TL was short and stabilized, and that the more unstable it became, the longer it also became. Also, the ellipse area was the area of the zone that was drawn with a trace line during the COP measurement. In this case, the C90 area, which corresponded to $90 \%$ of the ellipse area of the swayed COP, was selected, meaning that the better the static balance was, the narrower the area, and the worse it became, the wider the area became. The dynamic balancing ability was measured by identifying the maximum leaning angles, and the sum was used as the resultant value for the dynamic balance (D'Hondt et al., 2011). The sum taken after the completion of the 6-week PNF exercise programs were higher than the baseline test result, indicating that the PNF exercise programs improved the subject's dynamic balance ability. Also, the TL and the C90A area were both improved compared to the baseline, meaning that the balancing ability of the patient improved. As mentioned earlier, the subject of the present study were diagnosed with scoliosis through $\mathrm{X}$-ray imaging. Her cervical and thoracic spines were slightly convex towards the right while her lumbar spines were arched towards the right, showing S-type scoliosis. With this, based on the basic principles and procedures of PNF exercise programs, the PNF exercise programs were executed considering the subject's postures, the diagonal pattern of PNF, and the PNF technique. The subject's postures during the exercise programs progressed from those with wider supporting planes to those with narrower ones. This was to prevent pain on the waist and to increase the stability of the exercise process (Table 2). Also, from week 1 to week 6 , the first routine of the programs were the drill, in which the subject, lying in a hook-like position, pushed her pelvises down onto the floor with the help of the therapist, while allowing the
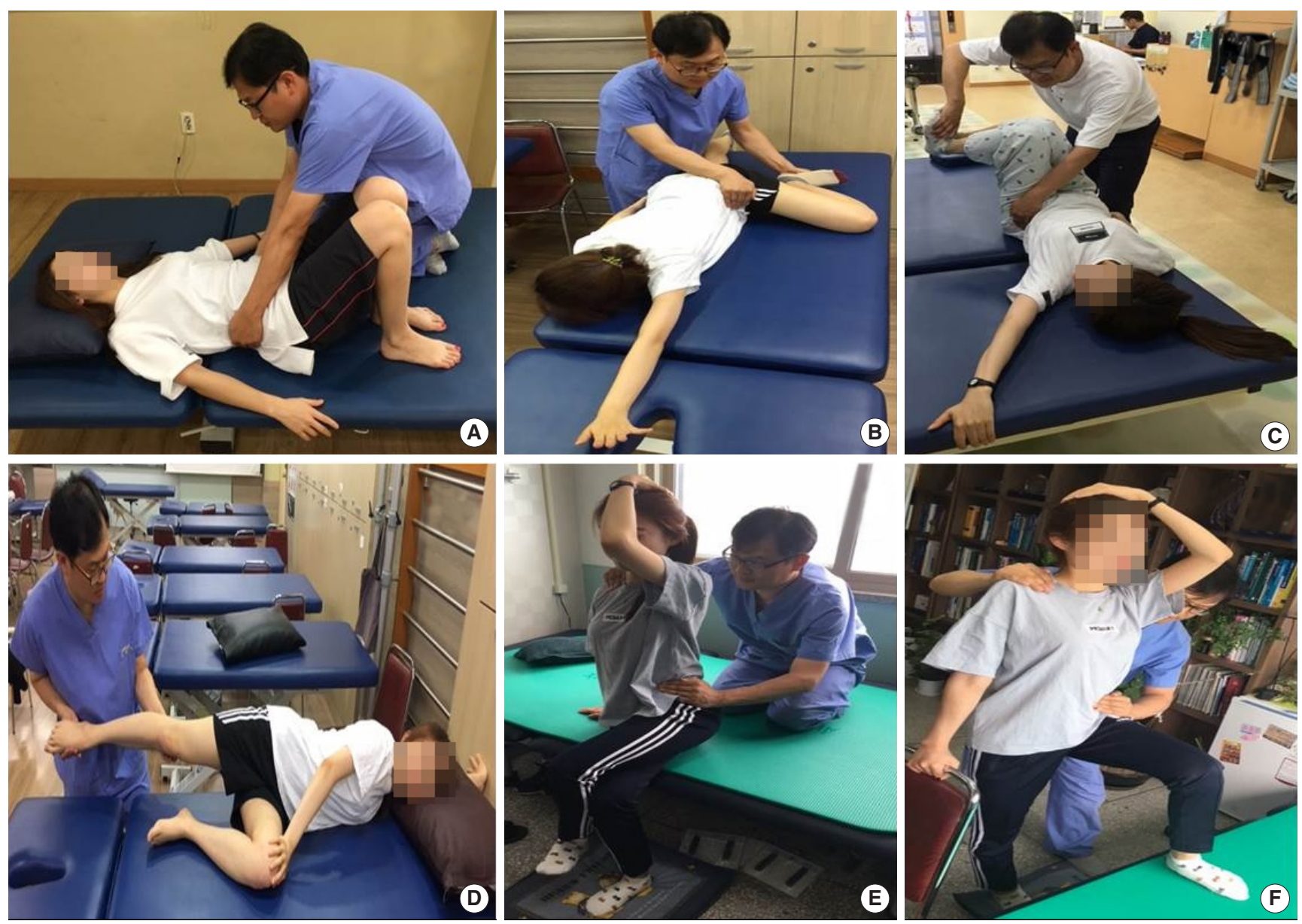

Fig. 4. Proprioceptive neuromuscular facilitation programs. (A) Stabilizing lumbo-pelvic systems; (B) Stabilizing lumbo-pelvic systems with correcting for spine alignment; (C) Correcting for spine alignment; (D) Correcting for spine alignment with extremities (CKCE); (E) Correcting for spine alignment with pelvis and extremities (CKCE); (F) Correcting for spine alignment with pelvis and extremities (CKCE). CKCE, closed kinetic chain exercise. 
abdominal muscles to contract (Fig. 4A). For this, the therapist held the iliac crest in both of his/her hands, and provided resistance to the front side while giving the subject a verbal command to push her pelvises towards the ground while trying posterior tilting at the same time. This reduced the subject's lumbar lordosis and increased the muscular force of her abdominal muscles. Kim et al. (2005) argued that the lumbar stabilization drill. (e.g., abdominal respiration) activated the transversus adominis, thus reducing her study subject's back pain. The second drill in the PNF exercise programs in this study involved the amphibian position, in which flexion-adduction-external rotation with knee flexion was performed with the left leg while the left arm performed flexion-abduction-external rotation to stabilize the lumbo-pelvic system and to elevate the scapular and pelvic bones on the left side, depressing the right-side scapular and pelvic bones so that the cervical and thoracic spines' arches towards the right and the lumbar spine's arches towards the left could be aligned to the normal positions. Here, to reduce the sway of the lumbar spine, the patterns were applied with the combination of isotonics, a PNF technique, allowing manual contact on the left leg (Fig. 4B). The third exercise was done in the supine position. Also, using both legs of each subject, the left-side pelvis was raised using combination of isotonics through the bilateral leg flexion pattern (Fig. 4C). In addition, the use of the pattern was limited to a narrow range, thus minimizing the burden of the exercise with resistance. The subject was also instructed to raise her left arms in flexion-abduction-external rotation not only to correct the lumbar spine abnormalities but also to improve the alignment of the cervical and thoracic spines. This is an exercise that "considers the whole person," part of the philosophy of PNF, which literally means considering the whole body of the patient. The fourth drill was executed in a side-lying position, where the right leg was doing extension-abduction-internal rotation while the right arm was doing flexion-abduction-external rotation in a closed-kinetic-chain exercise up to the upper wall to stretch the right arm of the patient (Fig. 4D). The combination of isotonics method was used to lower the right pelvis and to elevate the left pelvis, and while the extension-abduction-internal rotation on the right leg was being performed, the left leg and pelvis were doing flexion-abduction-external rotation to induce an anterior elevation pattern, rotating the pelvis of the patient towards the right side so that when seen from the back, the spinous process of the lumbar spine, which is turned towards the right, is restored to the center position by turning it towards the left (Fig. 1). According to Iwasaki et al. (2006), the closed-kinetic-chain exercise is mainly used to strengthen the muscles and increase their power, while the antagonists contract in an eccentric way to help stabilize the damaged joints. In their study, the contraction of the synergist was used to help the subject learn the exercise with ease. The fifth and sixth drills involved raising the posture of the patient so that the support base could be narrowed in the daily-life postures of sitting and standing (Fig. $4 \mathrm{E}, \mathrm{F})$. As the human body resembles a pendulum standing upside down, which is unstable and whose support base is narrow, it is difficult to achieve a balanced state. In the unstable posture where the support base is small, postural control requires much muscle power, for the movement. Moreover, the activity of the muscles is high (Dutt-Mazumder et al., 2016; Peterka and Black, 1990-1991). The lumbar spine, arched towards the right, and the pelvis, elevated on the right side, should be returned to the normal position by applying the anterior pattern on the left pelvis, along with the combination of isotonics. Moreover, the right arm of the patient is treated with extension-abduction-internal rotation so that the patient holds the bottom of the table and of the chair to lower the elevated right shoulder while aligning the thoracic spine, which is slightly arched towards the left. According to Zarzycka et al. (2009), exercise is a good way of treating idiopathic scoliosis patients, and among the various exercise methods, they proved that PNF is effective in correcting spinal deformity. In this study, the effectiveness of the PNF exercise programs in treating female scoliosis subject in the 20s was also verified.

This study was conducted over a period of 6 weeks, with the PNF exercise programs executed to enhance the alignment of the spines as well as the static and dynamic balance of the female idiopathic scoliosis subject in her 20s. The subject was a female in her 20s who was enthusiastic about her body shape. They confirmed that the PNF exercise programs for physical rehabilitation is effective in restoring the proper alignment of the spine and in improving the static and dynamic balancing abilities at the same time. Therefore, in the follow-up studies, more idiopathic scoliosis patients should be made to undergo the same PNF exercise programs to further demonstrate its effects. In addition, other topics for the follow-up studies may include quantitative suggestions on the manual resistance volume as well as the development of self-exercise programs based on the PNF exercise programs.

\section{CONFLICT OF INTEREST}

No potential conflict of interest relevant to this article was reported. 


\section{REFERENCES}

Adler S, Beckers D, Buck M. PNF in practice: an illustrated guide. 3rd ed. Berlin: Springer; 2002

Bae SS. A study of proprioceptive neuromuscular facilitation principles. J Korean Phys Soc 1993;5:109-114.

Borg FG, Laxåback G. Entropy of balance: some recent results. J Neuroeng Rehabil 2010;7:38.

Bunnell WP. The natural history of idiopathic scoliosis before skeletal maturity. Spine (Phila Pa 1976) 1986;11:773-776.

D’Hondt E, Deforche B, De Bourdeaudhuij I, Gentier I, Tanghe A, Shultz S, Lenoir M. Postural balance under normal and altered sensory conditions in normal-weight and overweight children. Clin Biomech (Bristol, Avon) 2011;26:84-89.

Dutt-Mazumder A, Challis J, Newell K. Maintenance of postural stability as a function of tilted base of support. Hum Mov Sci 2016;48:91-101.

Iwasaki T, Shiba N, Matsuse H, Nago T, Umezu Y, Tagawa Y, Nagata K, Basford JR. Improvement in knee extension strength through training by means of combined electrical stimulation and voluntary muscle contraction. Tohoku J Exp Med 2006;209:33-40.

Kim K, Park RJ, Bae SS. Effect of diaphragmatic breathing exercise on activation of trunk muscle of patients with low back pain. J Korean Soc Phys/Ther 2005;17:311-327.
Landauer F, Krismer M, Bauer R. Conservative treatment of idiopathic scoliosis. Orthopade 1997;26:808-817.

Nault ML, Allard P, Hinse S, Le Blanc R, Caron O, Labelle H, Sadeghi H. Relations between standing stability and body posture parameters in adolescent idiopathic scoliosis. Spine (Phila Pa 1976) 2002;27:19111917.

Pasha S, Capraro A, Cahill PJ, Dormans JP, Flynn JM. Bi-planar spinal stereoradiography of adolescent idiopathic scoliosis: considerations in 3D alignment and functional balance. Eur Spine J 2016;25:3234-3241.

Peterka RJ, Black FO. Age-related changes in human posture control: motor coordination tests. J Vestib Res 1990-1991;1:87-96.

Piirainen JM, Linnamo V, Cronin NJ, Avela J. Age-related neuromuscular function and dynamic balance control during slow and fast balance perturbations. J Neurophysiol 2013;110:2557-2562.

Srivastava A, Bayley E, Boszczyk BM. The management of high-grade spondylolisthesis and co-existent late-onset idiopathic scoliosis. Eur Spine J 2016;25:3027-3031.

Yamada K, Ikata T, Yamamoto H, Nakagawa Y, Tanaka H. Equilibrium function in scoliosis and active corrective plaster jacket for the treatment. Tokushima J Exp Med 1969;16:1-7.

Zarzycka M, Rozek K, Zarzycki M. Alternative methods of conservative treatment of idiopathic scoliosis. Ortop Traumatol Rehabil 2009;11: 396-412. 